\title{
Live weight and reproduction performance of Zimbabwean Blue and South African Black ostriches
}

\author{
S.W.P. Cloete ${ }^{1,2 \#}$, M.M. Brand ${ }^{1,3}$, L.C. Hoffman ${ }^{1}$ and M. Muller ${ }^{3}$ \\ ${ }^{1}$ Department of Animal Sciences, University of Stellenbosch, Private Bag X1, Matieland 7602, South Africa \\ ${ }^{2}$ Institute of Animal Production: Elsenburg, Private Bag X1, Elsenburg 7607, South Africa \\ ${ }^{3}$ Department of Food Science, Private Bag X1, Matieland 7602, South Africa
}

\begin{abstract}
Data obtained from a pair-mated ostrich flock located at Oudtshoorn in South Africa were used to derive line differences for live weight and reproduction performance in sexually mature ostriches of the Zimbabwean Blue (ZB) and South African Black (SAB) strains during 2003 to 2006. At the commencement of breeding ZB breeding stock were, on average, between 9 and 13\% heavier than SAB contemporaries. At the cessation of breeding the superiority of $\mathrm{ZB}$ birds was reduced to between 4 and $8 \%$, expressed relative to SAB breeding stock. Live weight at the commencement of breeding was complicated by an interaction between sire line and year. The interaction resulted from no line differences between SAB and ZB males in 2003, contrasted to marked differences in subsequent years. Egg production was affected by dam line, but not by the line of the sire or the interaction between dam line and sire line. Egg production of SAB females was almost twice that of ZB contemporaries, at respectively 43.3 and 23.3 eggs per season. The number of infertile eggs was not affected by any of the independent variables considered. The number of shell deaths was affected by both sire line and dam line. Overall, SAB females sustained lower levels of shell deaths than ZB females, while the eggs produced by mates of SAB males had higher shell deaths than mates of ZB males. Chick production was affected by dam line; the effects of sire line and the dam line x sire line interaction being non significant. Overall, SAB females produced more than double the number of chicks produced by their ZB contemporaries, at respectively 23.1 and 10.6 chicks per season. Both egg production and chick production were affected by a dam line $\mathrm{x}$ year interaction. No dam line difference was observed during 2003, whereas SAB females clearly outperformed ZB females in subsequent years. Further studies on the ostrich bloodlines and their crosses are needed to devise a selection and crossbreeding strategy for improving production and profitability in the industry.
\end{abstract}

Keywords: Ratite, chick production, egg production, mature live weight, crossbreeding

\#Corresponding author. E-mail: schalkc@elsenburg.com

\section{Introduction}

Research on the extensive breeding of common domesticated species such as poultry, cattle, pigs and small ruminants, has been done for a number of decades. Genetic and crossbreeding parameters, as well as line and breed differences, are thus readily available for these livestock species. Access to this information facilitates structured breeding programmes, involving line and crossbreeding and exploiting sexual dimorphism and heterosis. However, such information is severely limited or totally lacking in the ostrich industry (Cloete et al., 2002; Brand et al., 2005). It is thus impossible to provide the ostrich industry with definite guidelines as far as breed or strain differences are considered (Petitte \& Davis, 1999; Cloete et al., 2002). Observations made by Jarvis (1998) suggested considerable variation in mature live weight of the various strains of ostriches that are available for commercial production. Subsequent research suggested that Zimbabwean Blue ostriches (ZB Struthio camelus australis) are heavier than South African Blacks (SAB - S. c. var. domesticus) at the same age (Essa \& Cloete, 2006; Hoffman et al., 2007). In contrast, preliminary results suggested that SAB ostriches have a distinct advantage over ZB birds in terms of reproduction rate (Brand et al., 2005).

Several authors have suggested that the crossbreeding of different ostrich bloodlines could improve overall performance. According to Cloete et al. (2002), research into the crossbreeding of various lines constituting the current commercial ostrich population is urgently needed. At present, there is a tendency to 
crossbreed Kenyan Redneck (Struthio camelus massaicus), ZB and SAB ostriches without scientific evidence to guide crossbreeding decisions (Petitte \& Davis, 1999). It is contemplated that appropriate selection and culling strategies, together with crossbreeding, could improve the performance of the current and future flocks (Bunter et al., 2001a). Preliminary results suggest that the relative size advantage of ZB ostriches is compromised to an extent by poor chick survival (Essa \& Cloete, 2006). However, the progeny of ZB males and SAB females had a similar survival rate to purebred SAB birds, while they clearly outperformed the latter strain in terms of growth rate (Essa \& Cloete, 2006).

It is a prerequisite that the relative performance of strains or lines is known before a viable crossbreeding enterprise is planned. This study updates estimates for live weight and reproductive traits (including eggs produced, infertile eggs, dead-in-shell eggs, eggs not incubated as well as chicks produced) by ostrich males and females consisting of ZB and SAB ostriches.

\section{Materials and Methods}

Experimental animals used in the study were SAB and ZB birds from the commercial ostrich breeding flocks at the Oudtshoorn Research Farm (ORF) near Oudtshoorn, South Africa. The commercial SAB population has long been available on the experimental farm (Van Schalkwyk et al., 1996), while a population of ZB has recently been acquired from commercial producers (Brand et al., 2005). These birds were introduced to the experimental farm during March 2003, about two months prior to being joined in a pair-breeding structure on 28 May 2003. The birds were joined according to a structured breeding programme, involving the two pure strains and the reciprocal cross between them. One hundred and nine SAB breeding pairs and $12 \mathrm{ZB}$ breeding pairs represented the purebred combinations. The crossbred combination involving SAB males mated to ZB females was represented by 13 breeding pairs, while 24 breeding pairs represented ZB males mated to SAB females. Breeding ceased on 27 January 2004, when the birds were taken out of the breeding paddocks. The same basic outlay was followed during 2004-2006. The number of breeding pairs for the purebred combinations did not remain constant for the subsequent breeding seasons, as some birds were lost or incapacitated for breeding while new young breeders were introduced each year. However, it was ensured that each combination was represented by adequate numbers throughout. Only animal-year data of individuals present at the commencement and cessation of breeding were considered for statistical analysis of live weight data, while reproduction traits were only assessed in breeding females that were paired off for the entire season of eight months (June to January). Pairs that were disrupted by the death or incapacitation of a member were thus excluded when reproduction was assessed.

The same standard procedure was followed for the management of breeding pairs. During the off-season (February to May) the two different bloodlines of ostriches were maintained together in single sex flocks that had access to lucerne (Medicago sativa) pastures. The birds also had ad libitum access to gender specific diets of hammer-milled lucerne with a mineral-vitamin premix. Routine management included the harvesting of the white plumage (clipping and plucking). A standard drenching, vaccination and ectoparasite treatment was applied to all breeding birds. Birds were flushed two weeks prior to the breeding season with strategic dietary supplements (Lambrechts, 2004). Breeding pairs were housed in paddocks of ca. 0.25 ha. Paddocks were situated in the same area on the ORF. The typography is rolling with isolated shrubs and a rocky surface. Foraging material in the paddocks was limited and was consumed shortly after the commencement of breeding. No artificial shelter was supplied. All birds received the same breeder diet (8.5 MJ metabolisable energy and 120 g protein $/ \mathrm{kg}$ dry matter) at a level of $2.5 \mathrm{~kg}$ per bird per day throughout the entire breeding season, with free access to drinking water. Based on the preliminary results pertaining to the relative weight of the respective genotypes (Brand et al., 2005), it was decided to provide ZB pairs with $2.8 \mathrm{~kg}$ per day, and mixed pairs with $2.65 \mathrm{~kg} /$ day for the 2005 and 2006 breeding seasons. A standard procedure was followed for the management of eggs and chicks. Eggs were identified according to paddock number and date of production, and were collected in the evening on a daily basis. Egg production was recorded individually for each breeding paddock in the experiment. Eggs were transferred to the incubation facility in specially designed crates (to minimize transport and shock damage) immediately after collection. Eggs were disinfected through exposure to ultra-violet irradiation for $20 \mathrm{~min}$ in a UV disinfectant machine. After sanitation, eggs were stored for a maximum of six days in a cool room at $17{ }^{\circ} \mathrm{C}$ and $75 \%$ relative humidity, and were turned once daily through an angle of $45^{\circ}$. 
Eggs were stored upright with the air cell in the uppermost position. Eggs not suitable for incubation were noted for each breeding paddock in the experiment and removed from storage. There are various reasons for eggs being rejected, including: broken/cracked eggshells, chalky eggshells, loose air cells and too small eggs (Lambrechts et al., 2004). The cut-off point for too small eggs was $1-1.1 \mathrm{~kg}$.

The outcome of incubation for individual eggs (infertile, hatched, or dead in shell) was known (Van Schalkwyk et al., 2000; Bunter, 2002; Lambrechts, 2004). Buckeye ${ }^{\circledR}$, Prohatch ${ }^{\circledR}$ and Natureform ${ }^{\circledR}$ electronic incubators were used to artificially incubate the eggs for a period of 35 days at $36{ }^{\circ} \mathrm{C}$ and $28 \%$ relative humidity. Incubators were fogged weekly with F-10 for sanitation. Eggs were candled on day 21 to establish early embryonic deaths and fertility, and on day 35 to establish late embryonic deaths. Eggs showing no macroscopic development were regarded as infertile, and those with embryonic development that had ceased as embryonic deaths. After 35 days of incubation, eggs were transferred to the hatching unit, operated at $38.5{ }^{\circ} \mathrm{C}$ and $28 \%$ relative humidity. Here eggs were held vertically with no turning during the last week of incubation. Internal pipping generally commenced on the $41^{\text {st }}$ day of incubation, with external pipping some 6 - $12 \mathrm{~h}$ later. Eggs were transferred to separate compartments after external pipping to prevent loss of chick identity. Chicks were kept in the hatcher for $24 \mathrm{~h}$ after hatching to allow sufficient time for the navel to close and for the chick to dry off before being dispatched to the chick rearing facility at the ORF. The number of unhatched eggs and the reasons for not hatching during incubation were recorded. All data recorded were used to determine overall egg and chick production, as well as the average percentages of infertile eggs and shell deaths for individual pairs.

Individual live weights $(\mathrm{kg})$ were recorded for each bird at the commencement and cessation of breeding. Definitions for the reproductive traits were as follows:

- The number of clutches, a clutch being a sequence of eggs laid within four days (Bunter et al., 2001a)

- The time taken to commence laying after the formation of breeding pairs (Bunter et al., 2001a)

- The duration of lay, or time between first and last recorded egg of the season (Bunter et al., 2001a)

- Total egg production: total number of eggs produced per breeding season (Lambrechts et al., 2004)

- Number of infertile eggs for individual pairs (Bunter et al., 2001a)

- Number of dead-in-shell eggs for individual pairs (Bunter et al., 2001a)

- Number of eggs not incubated for reasons as set out previously (Lambrechts et al., 2004)

- Total chick production: number of day old chicks hatched (Lambrechts et al., 2004).

Live weight data of both sexes were assessed by least squares procedures (Harvey, 1990), with the effects of bloodline (SAB or ZB), year (2003 to 2006) the bloodline x year interaction included as fixed effects. Similar analyses were conducted on reproduction data, with sire line, dam line and year as main effects. Two-factor interactions between these main effects were also computed. Number of eggs produced was included as a linear covariate in the analysis on the number of eggs not incubated. Number of eggs set was accordingly included as a covariate in analyses on the number of infertile and dead-in-shell eggs, to adjust means for differences in egg production. Interactions involving year were only presented when significant for either live weight or reproduction data $(\mathrm{P}<0.05)$. The random effect of animal within bloodline was included in all cases to account for the repeated recording of animals in subsequent years. The variance components derived from these analyses allowed the calculation of repeatability of the traits recorded.

\section{Results and Discussion}

Repeatability estimates ( \pm s.e.) for live weight ranged from $0.63 \pm 0.03$ to $0.71 \pm 0.03$. Estimates for quantitative reproduction traits varied between $0.33 \pm 0.05$ and $0.37 \pm 0.04$. These estimates are consistent with those available in the literature (Van Schalkwyk et al., 1996; Cloete et al., 1998) and are not considered further.

At the commencement of breeding ZB males were heavier $(\mathrm{P}<0.01)$ than SAB males, the overall difference amounting to $8.2 \%$ (Table 1). At the cessation of breeding ZB males were still heavier than SAB males $(\mathrm{P}<0.05)$, but the difference amounted to only $4.2 \%$ in this instance. Zimbabwean Blue females were heavier ( $\mathrm{P}<0.01$ ) than SAB females, the differences amounting to $11.5 \%$ at the commencement of breeding and $8.6 \%$ at the cessation of breeding (Table 2). These results are consistent with a preliminary study on the same 
resource, but with only two years of data (Brand et al., 2005). The results are also in accordance with the fact that the body weight of ZB ostriches is expected to be larger than in SAB ostriches (Jarvis, 1998). The latter study did not state the sex and age of the ostriches used, but ZB ostriches were shown to have a larger body weight $(125 \mathrm{~kg})$ in comparison to SAB ostriches $(115 \mathrm{~kg})$, a difference of $9 \%$ in favour of the ZB genotype. Live weight of purebred SAB females in the present study corresponded well with other reports in the literature. These include a mean live weight of $115 \mathrm{~kg}$ at the commencement and $111 \mathrm{~kg}$ at the cessation of breeding (Lambrechts, 2004). Lambrechts et al. (1998) also reported that SAB females weighed $120 \mathrm{~kg}$ at the commencement of breeding. No comparable results could be found in the literature for mature ZB female ostriches.

Table 1 Least squares means ( \pm s.e.) for live weight $(\mathrm{kg})$ of male and female SAB and ZB ostriches at the commencement or cessation of breeding

\begin{tabular}{lccc}
\hline Gender and time of the breeding season & SAB & Bloodline & Significance \\
\hline Male birds & & & \\
$\quad$ Number of observations & 588 & 136 & $* *$ \\
$\quad$ Start & $123.4 \pm 1.4$ & $133.6 \pm 2.5$ & $*$ \\
$\quad$ End & $115.4 \pm 1.3$ & $120.2 \pm 2.3$ & \\
Female birds & 596 & 92 & $* *$ \\
$\quad$ Number of observations & $117.2 \pm 1.3$ & $130.6 \pm 2.6$ & $* *$ \\
$\quad$ Start & $103.2 \pm 1.1$ & $112.0 \pm 2.2$ & \\
$\quad$ End & & & \\
\hline
\end{tabular}

* - Significant $(\mathrm{P}<0.05)$; ** - Significant $(\mathrm{P}<0.01)$

Live weight at the commencement of breeding was complicated by a significant $(\mathrm{P}<0.01)$ interaction between sire line and year of production (Figure 1). No significant difference was found between sire lines during 2003 (120.4 \pm 2.8 vs. $122.8 \pm 3.7 \mathrm{~kg}$ for SAB and ZB males respectively; P >0.05). However, ZB males were between 9.1 and $12.7 \%$ heavier than $\mathrm{SAB}$ males during the subsequent years $(\mathrm{P}<0.01)$.

No evidence of a dam line $\mathrm{x}$ year interaction was present for initial live weight in females, the superiority of ZB females relative to SAB females ranging from $10.4 \%$ in 2005 to $12.6 \%$ in $2003(\mathrm{P}<0.01)$. The observed sire line $\mathrm{x}$ year interaction was also pointed out by Brand et al. (2005) in a preliminary study involving only the 2003 and 2004 production years. The latter authors suggested that it could result from the males being treated differently (compared to the females) before being introduced to ORF. A total of 23 males were obtained from one producer and 11 from another producer, whereas all the females were obtained from the latter producer. During subsequent years, after all birds were maintained under the same conditions for approximately 1.5 years, such differences would have been eliminated. This theory is supported by the relatively constant difference in favour of the ZB bloodline in females (all years) and males during 2004 - 2006. It can thus be argued that mature ZB birds are between 9 and 13\% heavier than SAB contemporaries at the commencement of breeding. The line difference appears to be less marked at the cessation of breeding, ranging from $4.2 \%$ in males to $8.6 \%$ in females. 


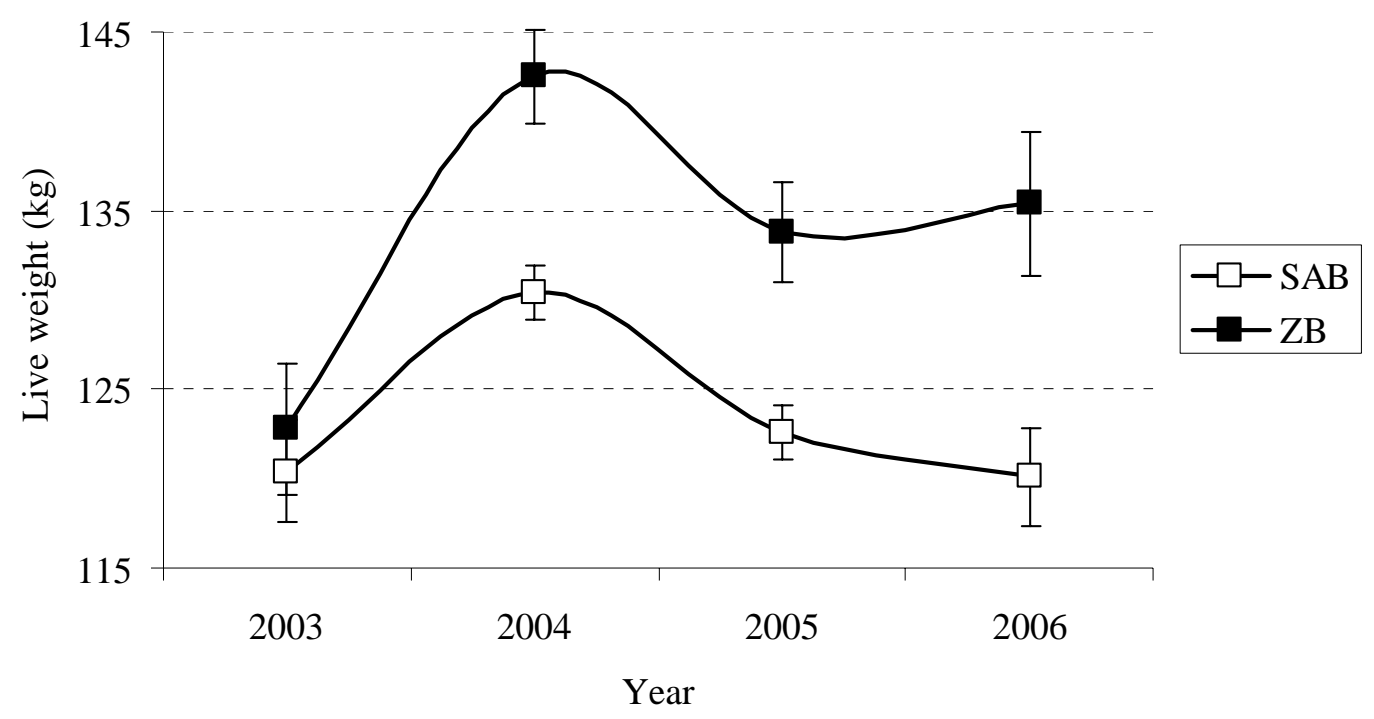

Figure 1 The interaction between sire line and year for live weight (kg) of South African Black (SAB) and Zimbabwean Blue (ZB) ostriches at the commencement of breeding. Vertical bars about the mean denote standard errors.

The traits number of clutches produced and the number of days from the commencement of breeding to the production of the first egg were independent of sire line, dam line and their interaction $(\mathrm{P}>0.25$; Table 2). The mean laying interval of ZB females was shorter than that of SAB females (136 \pm 9 vs. $172 \pm 5$ days respectively). Means for these traits are consistent with figures of 6.1 clutches per season, 41.7 days from the commencement of breeding to the first egg and a laying interval of 167 days reported by Cloete et al. (2005). Egg production was affected $(\mathrm{P}<0.01)$ by dam line, but not by sire line or the interaction between dam line and sire line. Overall, the egg production of ZB females amounted to only $54 \%$ of that produced by their SAB contemporaries $(23.3 \pm 3.6$ vs. $43.3 \pm 2.1$ eggs per season respectively). The close association of means for laying interval and egg production was expected, since they were reported to be governed by a largely similar set of genes (Cloete et al., 2005). This genotype difference is consistent with a $47 \%$ difference in favour of SAB females previously reported for a smaller sample of the same study population by Brand et al. (2005). The overall reproduction in the present study is somewhat lower compared to figures of respectively 33.6 and 49.5 eggs per season that were reported by Brand et al. (2005). This discrepancy possibly results from the inclusion of two-year-old females in the present study while they were excluded from the previous study, as two-year-old females are known to have a lower reproduction than older females (Cloete et al., 2006). Mean SAB performance in the present study was comparable to previous figures for egg production of 55.5 (Van Schalkwyk et al., 1996), 51.1 (Bunter et al., 2001a), 46.3 (Cloete et al., 2004), 46.3 (Lambrechts, 2004) and 44.7 (Cloete et al., 2005).

The number of infertile eggs produced by all bloodline combinations was fairly constant at 6 - 10 eggs, and was not affected $(\mathrm{P}>0.40$ ) by any of the independent variables considered (Table 2$)$. The number of dead-inshell chicks was affected $(\mathrm{P}<0.05)$ by both dam line and sire line. Overall, SAB females produced fewer deadin-shell chicks than ZB females (8.03 \pm 0.52 vs. $9.80 \pm 0.92$ respectively), when adjusted for the number of eggs set. The mates of SAB males correspondingly had a higher number of dead-in-shell chicks than the mates of ZB males (10.70 \pm 0.96 vs. $7.13 \pm 0.97$ respectively). Brand et al. (2005) reported similar differences in a smaller study. The seemingly robust results from both studies cannot be readily explained, and further studies are indicated. The number of eggs not incubated was affected by a significant sire line $\mathrm{x}$ dam line interaction after adjustment for the total number of eggs produced ( $\mathrm{P}<0.05$; Table 2 ). This interaction appeared to be associated 
with higher egg wastage in the crossbred combinations, and particularly in the SAB male by ZB female combination. This result was unexpected, and is poorly understood at present. A corresponding result was found by Brand et al. (2005), using a smaller data set, so the result appears to be quite robust. Chick production was only affected by dam line, the effects of sire line and the dam line $\mathrm{x}$ sire line interaction not being significant ( $\mathrm{P}>0.60$ ). Overall, the chick production of ZB females amounted to only $46 \%$ of that produced by their SAB contemporaries in a season $(10.6 \pm 2.5$ vs. $23.1 \pm 1.5$ chicks respectively; $\mathrm{P}<0.01)$. The corresponding means reported previously by Brand et al. (2005) were respectively 14.7 and 27.0 chicks per season for the respective genotypes. The somewhat lower means in the present study compared to those reported by Brand et al. (2005) could possibly also be ascribed to the inclusion of 2-year-old breeders, as argued previously. Comparable means found in the literature for chick production of SAB females were 29.1 (Van Schalkwyk et al., 1996), 23.8 (Bunter et al., 2001a), 22.9 (Cloete et al., 2004), 22.9 (Lambrechts, 2004) and 22.1 (Cloete et al., 2005). These figures are all in accordance with the present results. No comparable figures for ZB females could be obtained from the literature, with the exception of those reported by Brand et al. (2005).

Table 2 Least square means ( \pm s.e.) depicting the influence of sire (S) line (SAB or ZB) and dam (D) line (SAB or ZB) on female reproduction traits

\begin{tabular}{|c|c|c|c|c|c|c|c|}
\hline \multirow{2}{*}{$\begin{array}{r}\text { Sire line (S) } \\
\text { Dam line (D) }\end{array}$} & \multicolumn{2}{|c|}{ SAB } & \multicolumn{2}{|c|}{ ZB } & \multicolumn{3}{|c|}{ Significance } \\
\hline & SAB & ZB & SAB & ZB & $\mathrm{S}$ & $\mathrm{D}$ & $S \times D$ \\
\hline $\begin{array}{l}\text { Number of } \\
\text { observations (n) }\end{array}$ & 483 & 42 & 87 & 44 & & & \\
\hline $\begin{array}{l}\text { Number of } \\
\text { clutches (n) }\end{array}$ & $5.67 \pm 0.96$ & $6.76 \pm 0.75$ & $6.15 \pm 0.99$ & $5.36 \pm 0.73$ & 0.62 & 0.74 & 0.30 \\
\hline $\begin{array}{l}\text { Laying interval } \\
\text { (days) }\end{array}$ & $161 \pm 18$ & $144 \pm 14$ & $184 \pm 19$ & $128 \pm 14$ & 0.83 & $* *$ & 0.22 \\
\hline $\begin{array}{l}\text { Days to first egg } \\
\text { (days) }\end{array}$ & $32.3 \pm 11.3$ & $49.4 \pm 8.8$ & $52.7 \pm 11.8$ & $47.1 \pm 8.6$ & 0.38 & 0.29 & 0.28 \\
\hline $\begin{array}{l}\text { Egg production } \\
\text { (n) }\end{array}$ & $46.4 \pm 6.5$ & $28.3 \pm 5.7$ & $40.2 \pm 6.8$ & $18.3 \pm 5.6$ & 0.16 & $* *$ & 0.74 \\
\hline $\begin{array}{l}\text { Infertile eggs } \\
\text { (n) }\end{array}$ & $9.42 \pm 3.23$ & $6.65 \pm 2.55$ & $7.57 \pm 3.38$ & $7.80 \pm 2.48$ & 0.90 & 0.44 & 0.60 \\
\hline $\begin{array}{l}\text { Dead-in-shell } \\
\text { eggs (n) }\end{array}$ & $9.24 \pm 1.79$ & $12.15 \pm 1.41$ & $6.82 \pm 1.87$ & $7.45 \pm 1.38$ & $*$ & $*$ & 0.47 \\
\hline $\begin{array}{l}\text { Eggs not } \\
\text { incubated (n) }\end{array}$ & $3.53 \pm 1.00$ & $6.74 \pm 0.78$ & $4.74 \pm 1.05$ & $3.37 \pm 0.76$ & 0.28 & 0.06 & $*$ \\
\hline $\begin{array}{l}\text { Chicks produced } \\
\text { (n) }\end{array}$ & $21.2 \pm 4.5$ & $10.6 \pm 4.0$ & $25.0 \pm 4.7$ & $10.6 \pm 3.9$ & 0.63 & $* *$ & 0.65 \\
\hline
\end{tabular}

Overall egg and chick production were affected by a significant interaction of dam line with year (Figures 2 and 3, respectively). It is evident that the average performance of the lines was similar during 2003, while SAB females clearly outperformed ZB females in subsequent years. The average egg production of ZB females amounted to $96 \%$ of that for SAB females during 2003, while it declined to between 39 and $45 \%$ of average SAB performance in subsequent years. Corresponding figures for chick production were $90 \%$ during 2003 , with a subsequent decline to between 26 and $36 \%$. The production of SAB females appeared to be fairly consistent across years, and the interaction seemed to be primarily driven by the contrasting performance of ZB females during 2003 in comparison to 2004 - 2006. A comparable tendency was found in the preliminary study by Brand et al. (2005). However, the interactions were not reported as it did not reach statistical significance with the smaller data set ( $\mathrm{P}=0.11$ for egg production and $\mathrm{P}=0.07$ for chick production). 


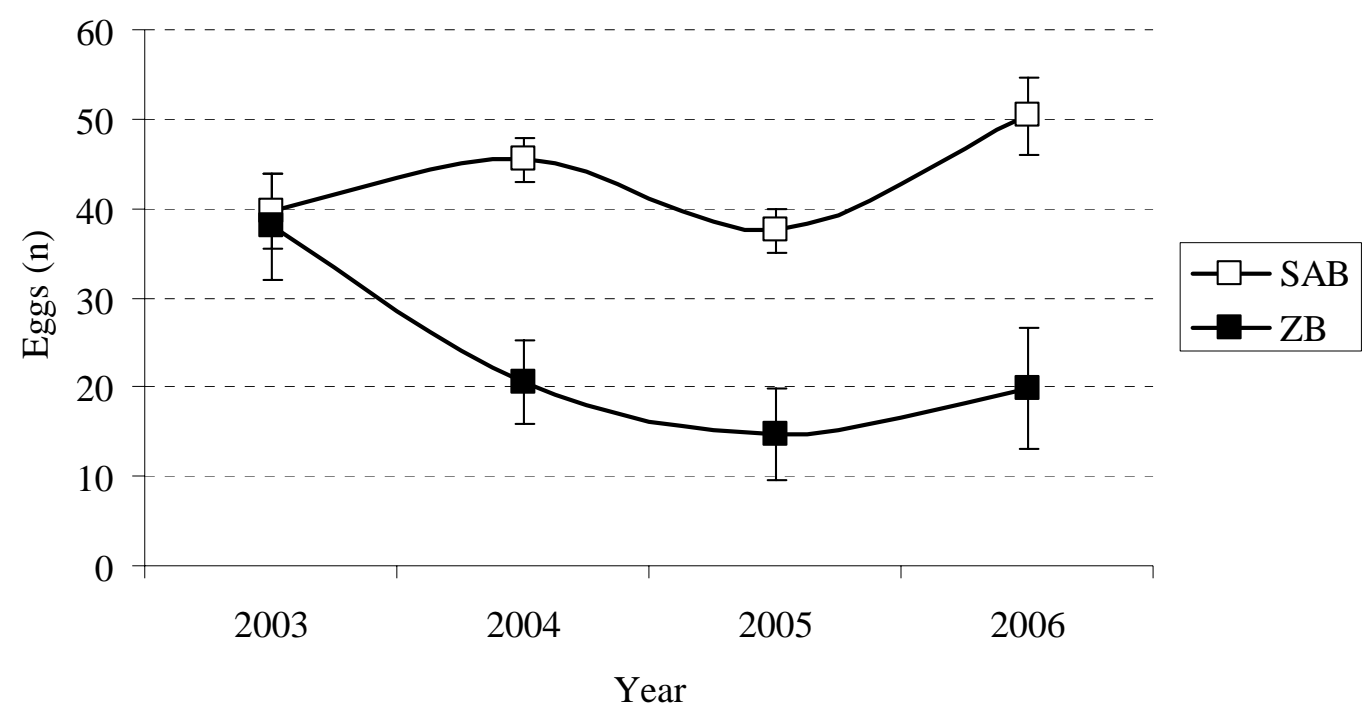

Figure 2 The interaction between sire line and year for egg production of South African Black (SAB) and Zimbabwean Blue (ZB) females over an 8-month breeding season. Vertical bars about the mean denote standard errors.

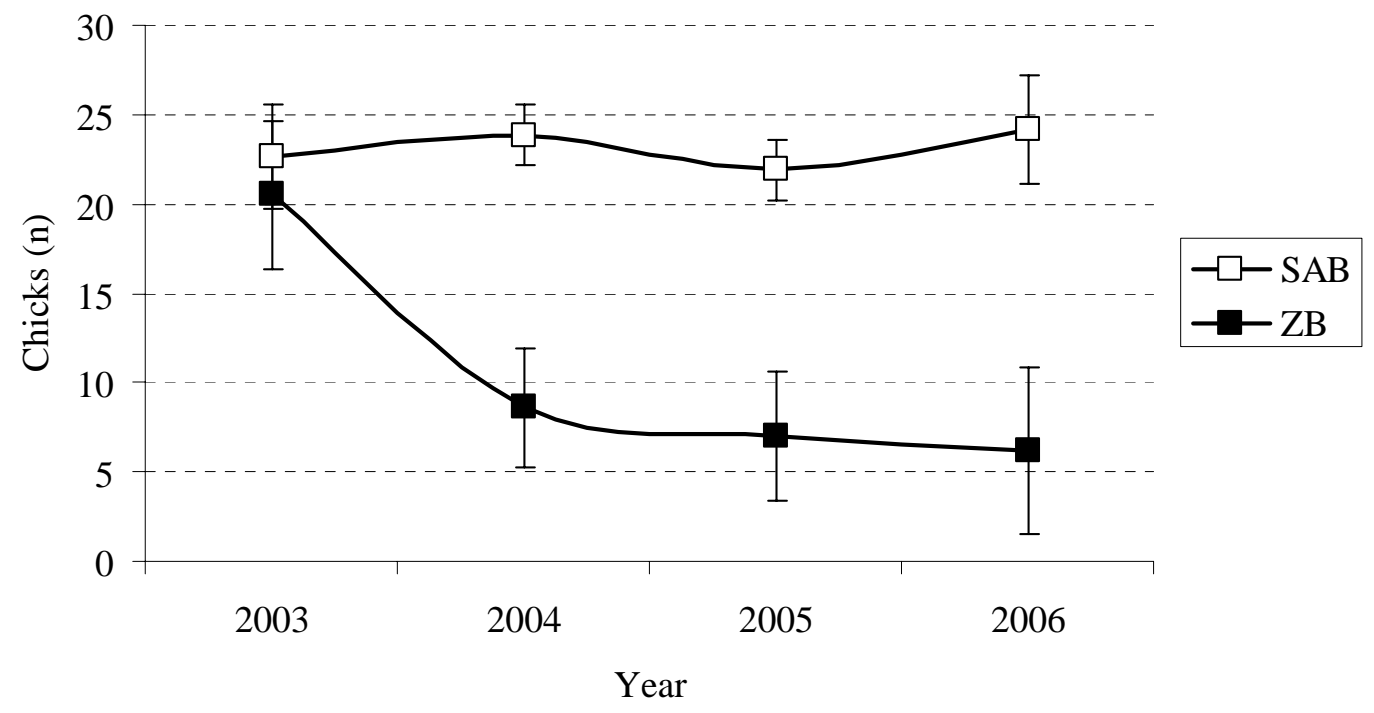

Figure 3 The interaction between sire line and year for chick production of South African Black (SAB) and Zimbabwean Blue (ZB) females over an 8-month breeding season. Vertical bars about the mean denote standard errors.

The lack of consistency in the production of ZB females is obscure and can only be speculated upon. A carry-over effect of nutritional treatments upon ostrich reproduction was described by Brand et al. (2002). However, it is considered unlikely that the on-farm treatment of the ZB females under commercial conditions prior to their introduction to the ORF could boost their performance during 2003 to the levels observed in 
Figures 2 and 3 as compared to 2004 - 2006. Since the ZB breeding stock were introduced to the ORF without details pertaining to age and pedigree, it is conceivable that some of these birds could have a fairly advanced age. It is known that ostrich egg and chick production of SAB females decline with an advanced age of older than 10 years (Bunter, 2002; Lambrechts, 2004). This decline is usually more evident in chick production than in egg production, apparently driven by a larger proportion of shell deaths in the eggs produced by older females (Brand et al., 2007). It is not known if similar trends should be expected in ZB breeding stock. Whereas the number of dead-in-shell chicks in the combination of SAB males with ZB females was unexpectedly high in the present study, no such effect was evident in the ZB females subjected to pure breeding. This result seems to be an indication against age being implicated in the observed trends. Apart from the preliminary study by Brand et al. (2005), we could not find previous studies reporting reproduction data for SAB and ZB ostriches to assist in the clarification of the obtained results. The present results could therefore not be validated against previous studies involving other genetic resources. The possible reasons for the observed interaction in the present study therefore remain unresolved, and further research is required to better understand the reproduction of the respective genotypes, and also to support or refute the results found in the present study.

\section{Conclusion}

In general, live weights of ZB ostriches were higher than those of SAB ostriches. The data at our disposal also suggest that egg and chick production of SAB females was likely to be higher than that of their ZB contemporaries. However, these results need to be revisited in future as the observed dam line $\mathrm{x}$ year interaction cast some doubt on the robustness of these findings.

The acceptance of the obtained results suggests that it would be viable to combine the relatively high live weight of $\mathrm{ZB}$ males with the relatively high reproduction of $\mathrm{SAB}$ females in a commercial crossbreeding operation. Preliminary studies on the productivity of progeny generated by this cross are positive, as survival of ZB x SAB chicks is enhanced to levels comparable to SAB chicks, and substantially better than those observed in purebred ZB chicks (Essa \& Cloete, 2006), while live weight and carcass yield are accordingly improved relative to purebred SAB progeny (Hoffman et al., 2007). Such a cross may also benefit from direct heterosis for chick growth and survival, which could not be quantified accurately because of a scarcity of data on crossbreeding in ostriches. Further studies on the relative reproduction performance of the two genotypes and their crosses are required to supplement these preliminary findings.

\section{Acknowledgements}

We express our sincerest gratitude to all those responsible for the maintenance and recording of the experimental flock at the Oudtshoorn Experimental farm. We are grateful to the Klein Karoo Cooperation for financial support and the usage of the SA Black resource flock, as well as the commercial producers entrusting us with their Zimbabwean Blue breeding stock. The study was partially funded by the THRIP programme of the South African Department of Trade and Industry.

\section{References}

Brand, M.M., Cloete, S.W.P., Hoffman, L.C. \& Muller, M., 2005. A comparison of live weights, body measurements and reproductive traits in Zimbabwean Blue ostriches (Struthio camelus australis) and South African Black ostriches (S. camelus var. domesticus). Proc. $3^{\text {rd }}$ Int. Ratite Sci. Symp. \& XII World Ostrich Congr., 14-16 ${ }^{\text {th }}$ October 2005. Ed: Carbajo, E., Madrid, Spain. pp. 73-80.

Brand, Z., Brand, T.S. \& Brown, C.R., 2002. The effect of dietary energy and protein levels during a breeding season of ostriches (Struthio camelus domesticus) on production the following season. S. Afr. J. Anim. Sci. 32, 226-230.

Brand, Z., Cloete, S.W.P., Brown, C.R, \& Malecki, I.A., 2007. Factors related to shell deaths during artificial incubation of ostrich eggs. J. S. Afr. Vet. Assoc. 78, 195-200.

Bunter, K.L., 2002. The genetic analysis of reproduction and production traits recorded for farmed ostriches (Struthio camelus). Ph.D. dissertation, University of New England, Armidale, Australia. 
Bunter, K.L., Cloete, S.W.P., Van Schalkwyk, S.J. \& Graser, H.-U., 2001a. Factors affecting reproductive performance in farmed ostriches. Proc. Assoc. Advmnt Anim. Breed. Genet. 14, 43-46.

Cloete, S.W.P., Bunter, K.L. \& Brand, Z., 2005. Genetic parameters for reproduction in ostriches. Proc. Assoc. Advmnt Anim. Breed. Gen. 16, 132-155.

Cloete, S.W.P., Bunter, K.L., Brand, Z. \& Lambrechts, H., 2004. (Co)variances for reproduction, egg weight and chick weight in ostriches. S. Afr. J. Anim. Sci. 34 (supplement 2), 17-19.

Cloete, S.W.P., Bunter, K.L., Lambrechts, H., Brand, Z., Swart, D. \& Greyling, J.P.C., 2006. Variance components for live weight, body measurements and reproductive traits of pair-mated ostrich females. $\mathrm{Br}$. Poult. Sci. 47, 147-158.

Cloete, S.W.P., Bunter, K.L. \& Van Schalkwyk, S.J., 2002. Progress towards a scientific breeding strategy for ostriches. Proc. $7^{\text {th }}$ World Cong. Gen. Appl. Livest. Prod. 30, 561-568. 18-23 August, Montpellier, France.

Cloete, S.W.P., Van Schalkwyk, S.J. \& Brand, Z., 1998. Ostrich breeding - progress towards a scientifically based strategy. Proc. $2^{\text {nd }}$ Int. Ratite Cong., 21-25 September 1998, Oudsthoorn. pp. 55-62.

Essa, F. \& Cloete, S.W.P., 2006. Survival and growth of purebred South African Black and Zimbabwean Blue ostriches and their reciprocal cross. Proc. $7^{\text {th }}$ World Cong. Gen. Appl. Livest. Prod., Bello Horizonte, Brazil. Communication 10-03.

Harvey, W.R., 1990. User's guide for LSMLMW and MIXMDL. PC-2 version, Mimeograph. 4255 Mumford drive, Columbus, Ohio 43220, USA.

Hoffman, L.C., Brand, M.M., Muller, M. \& Cloete, S.W.P., 2007. Carcass and muscle yield of ostriches as influenced by genotype. S. Afr. J. Anim. Sci. 37, 256-260.

Jarvis, M.J.F., 1998. The subspecies and races of ostriches and their present status in the wild. Proc. $2^{\text {nd }}$ Int. Ratite Cong., 21-25 September 1998, Oudtshoorn, South Africa, 4-8.

Lambrechts, H., 2004. Reproduction efficiency of ostriches in commercial farming systems. PhD dissertation, University of the Free State, South Africa.

Lambrechts, H., Cloete, S.W.P., Van Schalkwyk, S.J. \& Brand, Z., 1998. Phenotypic relationships of body measurements with egg production of 2-year old ostriches. Proc. $2^{\text {nd }}$ Int. Ratite Cong., 21-25 September 1998, Oudtshoorn, South Africa. pp. 72-74.

Lambrechts, H., Swart, D., Cloete, S.W.P., Greyling, J.P.C. \& Van Schalkwyk, S.J., 2004. The influence of stocking rate and male: female ratio on the production of breeding ostriches (Struthio camelus spp.) under commercial farming conditions. S. Afr. J. Anim. Sci. 34, 87-96.

Petitte, J.N. \& Davis, G., 1999. Breeding and genetics. In: The Ostrich: Biology, Production and Health. Ed. Deeming, D.C., UK, Oxon, Wallingford: CABI Publishing. pp. 275-292.

Van Schalkwyk, S.J., Cloete, S.W.P., Brown, C.R. \& Brand, Z., 2000. Hatching success of ostrich eggs in relation to setting, turning and angle of rotation. Br. Poult. Sci. 41, 46-52.

Van Schalkwyk, S.J., Cloete, S.W.P. \& De Kock, J.A., 1996. Repeatability and phenotypic correlations for live weight and reproduction in commercial ostrich breeding pairs. Br. Poult. Sci. 37, 953-962. 